\title{
Comparison of Intra Operative Cytology and Frozen Section with Permanent Pathologic Results in Ovarian Masses
}

\author{
Elaheh Ouladsahebmadarek*, Ali Dastranj Tabrizi, Manizheh Sayyah-Melli, Mehri Jafari-Shobeiri, \\ Parvin Mostafa-Garabaghi, Fatemeh Nazari
}

\begin{abstract}
Objectives: Making a decision on the extent of surgery for ovarian tumors depends on the intraoperative diagnosis. The aim of this study was to compare the intraoperative cytology and frozen section (FS) for diagnosis of ovarian masses.

Materials and Methods: One hundred and thirty-one patients with ovarian masses who underwent surgery for ovarian neoplasms were evaluated by intraoperative scrape and FS techniques and compared to final pathologic diagnoses.

Results: Scrape cytology for intraoperative diagnosis of benign ovarian tumors had sensitivity of $89.06 \%$ compared to $90.62 \%$ for FS. Specificity of both scrape and FS techniques for benign tumors was $94.91 \%$. Sensitivity and specificity of scrape for malignant ovarian tumors were $94.91 \%$ and $89.06 \%$, respectively. The related values for FS were $94.91 \%$ and $90.62 \%$. The overall accuracy percentage of scrape and FS for diagnosis of ovarian neoplasms was $91.86 \%$ and $92.68 \%$, respectively.

Conclusion: Intraoperative scrape cytology has high accuracy in diagnosis of ovarian tumors and is comparable with FS.

Keywords: Frozen sections, Intraoperative, Cytology, Pregnancy outcome
\end{abstract}

\section{Introduction}

Ovarian cancer is the 6th most common form of women's cancer worldwide (1). It accounts for $4 \%$ of all female cancers and $31 \%$ of cancers of the female genital tract. Ovarian cancer is the 4 th most common cause of death from malignancy in women (2). Almost it can be said that, the ovarian cancer has the widest pathologic variety between gynecological cancers and each pathology has a significant effect on the extent of surgery and follow up. The staging of ovarian cancer is totally surgical and determined by intraoperative findings, the extent of the disease and the potential sites of metastatic spread (3). Therefore, it requires accurate intra-operative diagnosis to prevent an extensive surgery in a young patient who desires to preserve her fertility or incurrence of a lengthy surgery to old patient, and also prevention of a second surgery after determination of final pathology.

For years, frozen section (FS) has been used to determine intraoperative diagnosis, but due to its limitations, methods such as fine needle aspiration (FNA) of ovarian masses, touch imprint and scraping and squash methods have been developed and underwent review to find a more rapid and more accurate alternative method for FS $(4,5)$. Over diagnosis of FS in malignant tumors is twice its' under diagnosis and under diagnosis of FS in borderline ovarian masses is multifold its over diagnosis (6).
In addition to the above mentioned facts, if a suspicious ovarian mass is suddenly discovered during surgery, preparation of FS is not available in many hospitals. So, we decided to investigate the scrape diagnostic method in evaluation of ovarian masses and detect its sensitivity, specificity and accuracy in our center compared to FS and final pathology.

\section{Materials and Methods \\ In a cross-sectional and descriptive-analytical study per- formed on women with ovarian masses who have been surgical candidates in Al-Zahra University dependent hospital during the years 2008 to 2011, sensitivity, speci- ficity and accuracy of scrape diagnostic method were eval- uated and compared to FS and permanent pathology. \\ In this period, 131 patients with ovarian masses were en- rolled into the study. After resection of the ovarian masses in the operating room they were immediately dispatched to pathology ward, fresh and without fixation, and grossly evaluated by pathologists. Gross details of the masses and pathologist's macroscopic diagnosis on the tumors were recorded. Then, mass surface was scrapped after cutting by scalpel and smeared on two glass slides. After one min- ute fixation in ethanol $96^{\circ}$, slides were stained with rap- id Hematoxylin and Eosin (HE) method. While staining cytology slides, FS test was performed on some pieces of}

Received 25 October 2014, Accepted 19 January 2015, Available online 13 February 2015

Women's Reproductive Health Research Center, Tabriz University of Medical Sciences, Tabriz, Iran.

*Corresponding author: Elaheh Ouladsahebmadarek, Women's Reproductive Health Research Center, Tabriz University of Medical Sciences,

Tabriz, Iran. Tel: +989141144132, Email: elmadarek33@yahoo.com 
the mass and then both slides of FS were evaluated and examined under microscope by pathologist. The result was reported to the operating room and type of surgery was determined according to the result. The slides of scraping were coded and evaluated after accomplishment of 131 patients by same pathologist. Then, results of the surgeon's primary guess on the type of mass, pathologist's macroscopic diagnosis, and results of scraping cytology and FS were compared with the permanent pathology as the gold standard to determine sensitivity, specificity, positive predictive value (PPV) and negative predictive value (NPV) for intraoperative scraping method and FS.

All data were analyzed using SPSS 14. Meanwhile sensitivity, specificity, PPV, NPV and accuracy for two intraoperative diagnostic methods were calculated.

\section{Scrape preparation method}

Impression method is applied for soft masses to smear on the glass slide, scraping method for masses with normal consistency and scratch and smear method for the fibrotic masses. The scrape slides were fixed in $96 \%$ ethylic alcohol for one minute. They were then stained by Hematoxylin and eosine $(\mathrm{H} \& \mathrm{E})$ in 2 minutes. All stages were performed by one pathologist.

\section{Ethical considerations}

This diagnostic method was performed parallel with the standard diagnostic method for patients and no changes were made in the type of treatment. No additional costs were charged on patients.

\section{Results}

From 131 patients included in this study 59 had malignant tumors, 64 had benign and 8 had borderline neoplasms according to the final pathology. Since our most important goal was to differentiate benign from malignant masses by two intraoperative methods, eight cases with diagnosis of borderline tumors were excluded from study. Demographic features of the patients and intra operative findings have been summarized in Tables 1 and 2. The surgeon could correctly identify $82.11 \%$ of ovarian lesions in operating room whereas the diagnosis of pathologist based on the gross of mass was accurate in $90.5 \%$.

Fifty-seven $(89.06 \%)$ of the benign neoplasms and 56 (91.86\%) of malignant tumors were accurately identified by scrape cytology (accuracy=94.91\%). FS accuracy for the diagnosis of ovarian neoplasms was $92.68 \%$ (Table 3). Sensitivity, specificity, PPV and NPV of scrape technique for diagnosis of benign tumors were $89.06 \%, 94.91 \%, 95 \%$ and $88.88 \%$, respectively. Correspondence values for FS were $90.62 \%, 94.91 \%, 90.62 \%$ and $94.91 \%$. The same values for malignant tumors have been shown in Figure 1.

The most common difficulty in diagnosis was in case of corpus luteoma, mucinous cyst and clear cell carcinoma, although serous type tumors, dysgerminoma and granulosa cell tumor were correctly diagnosed tumors by both methods.
Table 1. Demographic findings of patients

\begin{tabular}{lll}
\hline & Mean \pm SD & Range \\
\hline Age (year) & $43.12 \pm 14.74$ & $16-77$ \\
Gravida & $4.49 \pm 2.41$ & $0-12$ \\
Parity & $4.15 \pm 2.69$ & $0-12$ \\
Abortion & $4.1 \pm 0.78$ & $0-6$ \\
Menarche age (year) & $12.07 \pm 1.13$ & $11-18$ \\
Menopause age (year) & $49.79 \pm 4.37$ & $38-58$ \\
\hline
\end{tabular}

Table 2. Intraoperative findings

\begin{tabular}{lcccc}
\hline \multirow{2}{*}{ Ascites } & \multicolumn{4}{c}{ Ovarian mass } \\
\cline { 2 - 5 } & Unilateral & Cystic & Solid & Complex \\
\hline $35(28.45 \%)$ & $107(86.99 \%)$ & $61(50.4 \%)$ & $35(28.45 \%)$ & $27(21.95 \%)$ \\
\hline
\end{tabular}

Table 3. Correlation between final histological and intraoperative diagnoses

\begin{tabular}{llll}
\hline & \multicolumn{2}{c}{ Final histological diagnosis } & \multirow{2}{*}{ Total } \\
\cline { 2 - 3 } & \multicolumn{2}{c}{ Benign (\%) } & Malignant (\%) \\
\hline Scrape cytology diagnosis & & & \\
Benign & $57(89.06)$ & $3(5.08)$ & 60 \\
Malignant & $7(10.93)$ & $56(94.91)$ & 63 \\
Frozen section & & & \\
Benign & $58(90.62)$ & $3(5.08)$ & 61 \\
Malignant & $6(9.37)$ & $56(94.91)$ & 62 \\
\hline
\end{tabular}

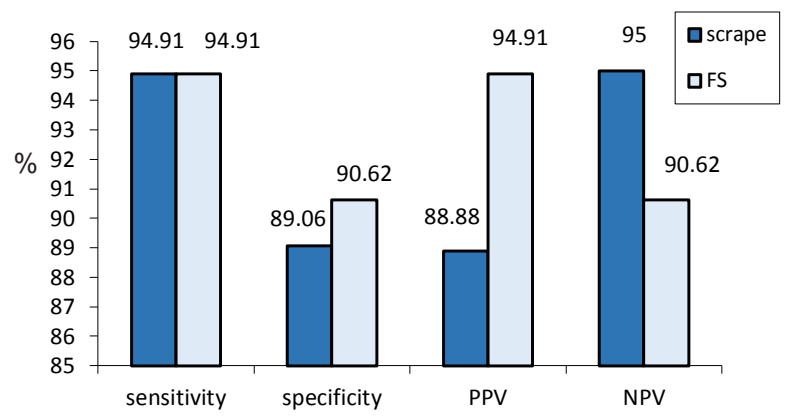

Figure 1. Sensitivity, specificity, PPV and NPV of scrape and frozen section for malignant ovarian tumors.

\section{Discussion}

Statistical review and analysis of the results indicate that close accuracy of scrape and FS methods is acceptable as compared to final pathology, and also, it is determined that sensitivity and specificity of scrape method is close to those of FS. As noted in Koss' book, high specificity of scrape method is predictable and desired and high PPV is expected (5). Khunamornpong and Siriaunkgul suggested in their study that scrape is actually helpful in differentiating between benign and malignant ovarian masses and especially, having a high predictive value in giving information on the probability of malignancy of tumor (7). The reported accuracy rate of FS for diagnosis of ovarian tumors by Suprasert et al. was $94 \%$ with sensitivities of $100 \%$ for diagnosis of benign, $84 \%$ for borderline and $92 \%$ for malignant tumors. The specificity rates for those 
tumors were $92.7 \%, 97.9 \%$, and $100 \%$, respectively (8). The results of Stewart et al. study showed that FS is more accurate than smear preparations; however cytology has a complementary role in the assessment of ovarian neoplasia providing a more specific diagnosis in some cases (9). It will even be more helpful if the results are combined with surgeon's intraoperative diagnosis. As for diagnosis of pathology types, comparing the results of scrape and FS with final pathology indicates that there were errors in diagnosis of pathology type in both methods, which were almost the same.

Inability to differentiate corpus luteoma and unacceptable reports in our study has been also found in the study by Khunamornpong and Siriaunkgul; he has declared the lack of adequate cells in samples, especially when lysis has occurred, and also in corpus luteoma as a cause of reduced accuracy of scrape (7).

The study of Stewart et al. revealed that accuracy of FS in diagnosis of clear cell carcinoma was 39\% (10). In our study, clear cell carcinoma had not been diagnosed by scrape and FS, but declared in the final pathology; this may be due to unsuitable staining of these tissues. According to Vrdoljak-Mozetic et al., this tumor is more diagnosable with Giemsa staining (11). Khunamornpong et al. suggested Diff-Quick staining for these masses (12).

Wasinghon et al. showed that FS diagnosis was a good approach for the surgical management of patients with ovarian masses (13). Moreover, Shidham et al (14) demonstrated that, for the best result, quality of preparation and staining of the slides should be according to the nature of the tissue; and according to Koss, the most important factor for accurate diagnosis is preparing suitable slides. Pathologic diagnostic mistakes about mucinous cysts observed in our study is also in accordance with the results by other researches confirming their theory that in order to increase the accuracy of intraoperative cytology, the existence of more cells on the slides and a structural tissue with more healthy cells is necessary (14).

In our study, possibility of correct diagnosis of a cystic ovarian mass with low cellularity rate was low. Also a similar result has been shown in the study by Lu et al., which reported high false negative rate $(35 \%)$ and low sensitivity (52\%) for intraoperative cytology with smearing slides by thin prep method of ovarian cyst fluid in diagnosis of malignant tumors; the overall tissue diagnosis accuracy in this method was $20 \%-25 \%$, and in our study, the most unacceptable results were about benign cystic masses or corpus luteoma (24.4\%) (15).

However, despite all the restrictions, in an emergency setting, the benignity or malignancy of the ovarian masses could be determined based on the result of scrape cytology. This conclusion should be taken more serious if malignant cells are found in cytology and so, the possibility of malignancy would be very high.

Besides, one should be always careful while operating based on intraoperative diagnostics, either scrape or FS, and the final decision should be made based on all the current circumstances.

\section{Conclusion}

Intraoperative cytology by scrape smears is easier, faster and more accessible than FS slides and with a high and comparable percentage of accuracy, sensitivity and specificity is a good alternative for FS.

\section{Ethical issues}

This study has been carried out with taking informed consent from patients for using different diagnostic techniques during surgery.

\section{Conflict of interests}

The authors declare that they have no conflict of interests.

\section{Acknowledgments}

We appreciate the cooperation of Women's Reproductive Health Research Center's staffs. This research was supported by Research Center (Grant No. 847).

\section{References}

1. Fleming GF, Seidman J, Zaino RJ, Rubin SC. Epithelial ovarian cancer. In: Barakat RR, Randall ME, editors. Principles and practice of Gynecologic Oncology. USA: Williams \& Wilkins; 2009. p. 763-835.

2. Berek JS FM, Hacker NF. Epithelial ovarian, fallopian tube and peritoneal cancer. In: Berek JS HN, editor. Berek \& Hacker's Gynecologic Oncology. USA: Lippincott, Williams \& Wilkins; 2010. p. 443-508.

3. Van JR, Gershenson DM. Ovarian cancer: Etiology, Screening, and Surgery. In: Rock JA, editor. TeLinde's operative gynecology. USA: Lippincott, Williams \& Wilkins; 2008. p. 1307-39.

4. Anastasiadis PG, Romanidis KN, Polichronidis A, Koutlaki NG, Tamiolakis D, Simopoulos K. The contribution of rapid intraoperative cytology to the improvement of ovarian cancer staging. Gynecol Oncol 2002;86(3):244-9.

5. Lejung BM. Techniques of fine-needle aspiration, smear preparation, and principles of interpretation. In: Koss LG, Melamed MR, editors. Koss's Diagnostic Cytology and it's Histopathologic Bases. USA: Lippincott; 2006. p. 1056-80.

6. Tempfer CB, Polterauer S, Bentz EK, Reinthaller A, Hefler LA. Accuracy of intraoperative frozen section analysis in borderline tumors of the ovary: a retrospective analysis of 96 cases and review of the literature. Gynecol Oncol 2007;107(2):248-52. doi: 10.1016/j.ygyno.2007.06.008.

7. Khunamornpong S, Siriaunkgul S. Scrape cytology of the ovaries: potential role in intraoperative consultation of ovarian lesions. Diagn Cytopathol 2003;28(5):250-7. doi: 10.1002/dc.10273.

8. Suprasert P, Khunamornpong S, Phusong A, Settakorn J, Siriaungkul S. Accuracy of intra-operative frozen sections in the diagnosis of ovarian masses. Asian Pac J Cancer Prev 2008;9(4): 737-40.

9. Stewart CJ, Brennan BA, Koay E, Naran A, Ruba S. Value of cytology in the intraoperative assessment of 
ovarian tumors: a review of 402 cases and comparison with frozen section diagnosis. Cancer Cytopathol 2010;118(3):127-36. doi: 10.1002/cncy.20073.

10. Stewart CJ, Brennan BA, Hammond IG, Leung YC, McCartney AJ, Ruba S. Intraoperative assessment of clear cell carcinoma of the ovary. Int J Gynecol Pathol 2008;27(4):475-82. doi: 10.1097/ PGP.0b013e31816b5cff.

11. Vrdoljak-Mozetic D, Stankovic T, Krasevic M, Versa-Ostojic D, Stemberger-Papic S, Rupcic S. Intraoperative cytology of clear cell carcinoma of the ovary. Cytopathology 2006;17(6):390-5. doi: 10.1111/j.1365-2303.2006.00373.x

12. Khunamornpong S, Thorner PS, Suprasert P, Siriaunkgul S. Clear-cell adenocarcinoma of the female genital tract: presence of hyaline stroma and tigroid background in various types of cytologic specimens. Diagn Cytopathol 2005;32(6):336-40. doi: 10.1002/dc.20257

13. Wasinghon P, Suthippintawong C, Tuipae S. The accuracy of intraoperative frozen sections in the diagnosis of ovarian tumors. J Med Assoc Thai 2008;91(12):1791-5.

14. Shidham V, Gupta D, Galindo LM, Haber M, Grotkowski C, Edmonds $\mathrm{P}$, et al. Intraoperative scrape cytology: comparison with frozen sections, using receiver operating characteristic (ROC) curve. Diagn Cytopathol 2000;23(2):134-9.

15. Lu D, Davila RM, Pinto KR, Lu DW. ThinPrep evaluation of fluid samples aspirated from cystic ovarian masses. Diagn Cytopathol 2004;30(5):320-4. doi: 10.1002/dc.20046

Copyright $(2015$ The Author(s); This is an open-access article distributed under the terms of the Creative Commons Attribution License (http://creativecommons.org/licenses/by/4.0), which permits unrestricted use, distribution, and reproduction in any medium, provided the original work is properly cited. 